\title{
¿Existen especies de NaVicula (Bacillariophyta) exclusivas de ambientes de alta montaña en Argentina?
}

\author{
CLAUDIA SEELIGMANN ${ }^{1}$ y NORA I. MAIDANA ${ }^{2}$
}

\begin{abstract}
Summary: Are there any Navicula species (Bacillariophyta) living exclusively in high altitude environments of Argentina? In order to analyze the geographical distribution of 28 species of the genus Navicula Bory sensu stricto, identified in high mountain wetlands in Argentina, we consulted the five available catalogs, publications not included in those works, and unpublished data collected in surveys conducted by our team. Information was gathered from more than 190 water bodies in Argentina, Bolivia, and Chile, from which maps were constructed using the DIVA-GIS software. These maps allowed us to visualize the distribution of species and relate them to the altitude of the sites where they were found. Only five taxa ( $18 \%$ of total identified species in high mountain wetlands) were found exclusively in high altitude water bodies in Argentina: Navicula atacamana, N. carvajaliana, N. parinacota, N. salinicola var. boliviana and $N$. phylleptosoma. Of these species, the only one that is not exclusively American is $N$. phylleptosoma, which would have a wide distribution. While there are numerous studies carried out on the subject of diatoms of Argentina, it is interesting to note the large area of the country that still remains to be surveyed. Future studies may modify the knowledge we now have regarding the distribution of species which is, apparently, restricted to high mountain environments.
\end{abstract}

Key words: Navicula, diatoms, biogeography, high mountain wetlands, Andes, South America.

Resumen: Con el objetivo de analizar la distribución geográfica de 28 especies del género Navicula Bory sensu stricto identificadas en humedales de alta montaña en Argentina, se consultaron cinco catálogos disponibles, publicaciones no incluidas en esas obras y datos recabados en relevamientos inéditos realizados por nuestro equipo de trabajo, considerando la nomenclatura actual y los sinónimos de cada especie. Se reunió información procedente de más de 190 cuerpos de agua de Argentina, Bolivia y Chile, a partir de la cual se construyeron mapas utilizando el software DIVA-GIS, que permitieron visualizar la distribución de las especies y relacionarla con la altura sobre el nivel del mar de los sitios donde fueron encontradas. Solo cinco especies (el $18 \%$ del total de especies identificadas en los humedales de alta montaña) fueron halladas exclusivamente en cuerpos de agua de altura de Argentina: Navicula atacamana, $N$. carvajaliana, $N$. parinacota, $N$. salinicola var. boliviana y $N$. phylleptosoma. De estas especies, la única que no es exclusivamente americana es $N$. phylleptosoma, que tendría una amplia distribución. Si bien hay numerosos trabajos realizados sobre diatomeas de Argentina, es interesante notar la gran superficie del país que aun resta por relevar. Es posible que futuros estudios modifiquen el conocimiento que ahora tenemos sobre la distribución de las especies aparentemente restringidas a ambientes de altura.

Palabras clave: Navicula, diatomeas, biogeografía, humedales de altura, Andes, Sudamérica.

\section{INTRODUCCIÓN}

Los Andes Centrales abarcan la región norte de Argentina y Chile, oeste de Bolivia y sur de

${ }^{1}$ ILINOA, Facultad de Cs. Naturales e Inst. M. Lillo. M. Lillo 205, 4000, S. M. de Tucumán, Argentina. cseeligmann@ hotmail.com

${ }^{2}$ Dpto. de Biodiversidad y Biología Experimental, Facultad de Cs. Exactas y Naturales, Universidad de Buenos Aires. C. Universitaria, 1428, CABA, Argentina.
Perú e incluyen uno de los complejos evaporíticos continentales más grandes del mundo (Ericksen \& Salas, 1989). El progresivo ascenso de los Andes, funcionó como una barrera climática que provocó una aridización en la Puna al no permitir la entrada de vientos húmedos y aisló los sistemas fluviales, generando cuencas cerradas que dieron lugar a la formación de salares (Alonso et al., 2006). En regiones montañosas, como la Puna, además de los salares existen otros tipos de humedales como lagos, lagunas, vegas, ríos, arroyos, etc. (según la 
definición de la Convención Ramsar de 1971), que también albergan una riquísima flora y fauna.

En Argentina, los numerosos y variados cuerpos de agua en alta montaña (a más de $2000 \mathrm{~m} \mathrm{snm}$ ) se encuentran, según el esquema biogeográfico de Morrone (2006), dentro de las provincias Páramo Norandina y de la Puna. Estas provincias, biogeográficamente muy relacionadas, pertenecen a la Zona de transición de América del Sur, como parte de la Región Andina. Fitogeográficamente, la región de estudio queda incluida dentro de las provincias de Prepuna, Puna y Altoandina (Cabrera \& Willink, 1973) y según Brown \& Pacheco (2006), dentro de las ecorregiones de Puna y Altos Andes.

De acuerdo con Morrone (2006) los patrones de distribución de un grupo específico o de una especie tienen en cuenta procesos históricos y ecológicos y la mayor parte de la biota de la Región Andina habría evolucionado en Patagonia y gradualmente se habría propagado hacia el norte durante el Terciario y Pleistoceno, hacia la Zona de transición de América del Sur.

Los lagos y lagunas de alta montaña, así como los de altas latitudes de condiciones muy frías, se encuentran sometidos a eventos climáticos que condicionan las características de sus aguas y por lo tanto su biota presenta adaptaciones especiales para estas condiciones de vida (Sánchez Castillo et al., 1989). Estos ambientes son naturalmente inestables a causa de la estacionalidad de las lluvias y sequías periódicas, tienen aguas alcalinas con alta conductividad y están sometidos a una fuerte radiación solar y vientos intensos. Según Morales \& Vis (2007), estos ecosistemas proveen grandes posibilidades para la presencia de especies endémicas y señalan que más de $20 \%$ de los taxones de diatomeas que identificaron en tres arroyos de las yungas de La Paz (Bolivia) no fueron hallados en otras partes del mundo. De la misma manera, en un análisis de muestras del Río Desagüadero (Bolivia), ubicado a $3700 \mathrm{~m}$ snm, Morales et al. (2012) encontraron un alto porcentaje de taxones desconocidos y uno bajo de cosmopolitas. Estos mismos autores, analizando los resultados de investigaciones realizadas en ecosistemas andinos por Rumrich et al. (2000), revelan un $42 \%$ de especies cosmopolitas y un $21 \%$ de posibles endemismos, incluyendo solo un $9,5 \%$ de especies nuevas, número que señalan como sorprendentemente bajo y que no permitiría caracterizar a la flora de diatomeas andina como exclusiva.

Como ya fue señalado por Maidana \& Seeligmann (2006), en la mayoría de los trabajos que se han publicado hasta finales del siglo XX, las identificaciones se hicieron utilizando las monografías disponibles, prácticamente todas ellas referidas a floras europeas y, en mucho menor grado, norteamericanas.

Rumrich et al. (2000), en su listado bibliográfico, mencionan escasísimos trabajos realizados por investigadores norte y sudamericanos por lo que aparentemente desconocerían la existencia de algunas especies previamente descriptas para la región. Por otro lado, autores como Spaulding \& McKnigth (1999) y Vanormelingen et al. (2008) consideran que es posible que el bajo porcentaje de especies exclusivamente andinas y el alto porcentaje de especies de regiones templadas de Europa que hallaron aquellos autores sea el resultado de forzar las identificaciones para asignarles nombres de taxones europeos.

Los ambientes de alta montaña son actualmente foco de interés ya que ofrecen bienes y servicios fundamentales, envisten un alto valor por su diversidad biológica y endemismos, constituyen refugios para aves migratorias lo que impulsó la creación de áreas protegidas y brindan interesantes oportunidades de diversificación económica a los grupos humanos asentados. Por otra parte desde el punto de vista arqueológico existen localidades cuya historia se remonta a la ocupación prehispánica.

En el Noroeste Argentino, desde 1998 comenzamos el estudio de las diatomeas de ambientes de altura de las provincias de Jujuy y Catamarca con el objetivo de evaluar su composición específica y su estructura comunitaria e indagar algunas relaciones entre este grupo de algas y las variables ambientales (Seeligmann \& Maidana, 2003; Maidana \& Seeligmann, 2006; Seeligmann et al., 2008; Maidana et al., 2009, 2011).

En los humedales de alta montaña estudiados en Argentina, hemos encontrado hasta el momento 28 especies de Navicula Bory, que fue uno de los géneros más diversos (Maidana et al., 2011). Las especies identificadas fueron Navicula atacamana Patrick, N. capitatoradiata Germain, $N$. carvajaliana Patrick, N. cari Ehrenberg, N. cincta (Ehrenberg) Ralfs, N. cryptocephala Kützing, $N$. cryptotenella Lange-Bertalot, $N$. cryptenelloides 
Lange-Bertalot, $N$. erifuga Lange-Bertalot, $N$. gregaria Donkin, $N$. lauca Rumrich \& LangeBertalot, N. libonensis Schoeman, N. microcari Lange-Bertalot, N. microdigitoradiata LangeBertalot, N. parinacota Rumrich \& Lange-Bertalot, $N$. peregrina (Ehrenberg) Kützing, $N$. perminuta Grunow in Van Heurck, N. phyleptosoma LangeBertalot, N. pseudogracilis Hustedt, N. radiosa Kützing, N. recens (Lange-Bertalot.) LangeBertalot, $N$. salinicola Hustedt var. salinicola, $N$. salinicola var. boliviana Patrick, N. tenelloides Hustedt, N. tripunctata (O. F. Müller) Bory, N. trivialis Lange-Bertalot, $N$. veneta Kützing y $N$. viridula (Kützing) Ehrenberg.

Esta riqueza de especies nos llevó a plantearnos una serie de interrogantes, como por ejemplo si es que alguna de esas especies es exclusiva de ambientes de alta montaña; por qué hay lagunas muy cercanas, con características similares, que no tienen la misma diatomoflora y con qué parámetros se puede relacionar esta distribución (la altura, el aislamiento o características físicoquímicas particulares de cada humedal).

El objetivo principal de este trabajo es buscar una respuesta a estos interrogantes, para lo cual analizamos la distribución en Argentina de las 28 especies antes mencionadas y confrontamos estos resultados con los obtenidos por otros autores que focalizaron sus estudios en ambientes ubicados en alta cota en los dos países limítrofes de Argentina con los que comparte este tipo de ambientes (Chile y Bolivia).

\section{Material y Método}

Los resultados analizados corresponden a muestreos que realizamos durante siete años, en los cuales se relevaron más de 60 cuerpos de agua de altura (>2000 m snm) y se identificaron 377 taxones infragenéricos de diatomeas (Seeligmann \& Maidana, 2003; Maidana \& Seeligmann, 2006; Seeligmann et al., 2008; Maidana et al., 2009, 2011).

Para conocer la distribución en Argentina de las 28 especies identificadas de Navicula, se consultaron los cinco catálogos publicados (Ferrario \& Galván, 1989; Luchini \& Verona, 1972; Martínez Macchiavello \& Salas - Arámburu, 1994; Tell, 1985; Vouilloud, 2003); publicaciones no incluidas en esas obras (Tabla 1) y datos recabados en relevamientos inéditos realizados por nuestro equipo. Asimismo, consultamos trabajos realizados en ambientes de alta montaña de Bolivia y Chile y algunas monografías que incluyen a los 3 países. En todos los casos tuvimos en cuenta la nomenclatura actual y los sinónimos de cada especie (VanLandingham, 1969-1979 y publicaciones posteriores de otros autores).

La mayoría de las identificaciones se corroboraron siguiendo a Lange-Bertalot (2001), excepto en los casos de especies exclusivamente americanas, en los que se consultó la bibliografía original. Esto pudo hacerse cuando se disponía de la información necesaria (descripción, dimensiones, ilustraciones). Sin embargo, un uso muy común en los trabajos ficoflorísticos de Argentina es incluir solamente descripciones y/o ilustraciones de las especies que resultaban nuevas citas para el país mientras que las restantes aparecían listadas sin mayores datos taxonómicos. En este tipo de trabajos, exceptuando los de nuestra autoría, fue imposible realizar un control de las identificaciones.

De esta manera, reunimos información procedente de más de 190 cuerpos de agua de Argentina y países limítrofes en los cuales se encuentran humedales de alta montaña (Fig. 1). De estos, en 175 se registró alguna de las 28 especies de Navicula encontradas por nosotros en ambientes de altura. Con los datos recabados construimos mapas (utilizando el software DIVA-GIS 7.5) que permiten visualizar la distribución de las especies según la bibliografía consultada y relacionarla con, por ejemplo, la altura sobre el nivel del mar de los sitios donde fueron encontradas y la salinidad de los ambientes de alta montaña de Argentina en los que fueron hallados.

\section{Resultados}

De las 28 especies de Navicula identificadas en los humedales de alta montaña de Argentina, las que tuvieron la mayor frecuencia de citas sobre el total de los lugares relevados en este trabajo (Tabla 2, Fig. 1) fueron $N$. veneta $(29 \%), N$. lauca $(26 \%), N$. radiosa (25\%), N. cryptotenella, N. gregaria y N. salinicola var. salinicola ( $23 \%$ cada una) y $N$. cryptocephala (21\%); las restantes no alcanzaron el 20\% (Fig. 2). Por otra parte, solo cinco de estos taxones fueron 
Bol. Soc. Argent. Bot. 48 (3-4) 2013

Tabla 1. Categorización de la bibliografía consultada para la distribución de las especies de Navicula por países y tipo de publicaciones.

\begin{tabular}{|c|c|c|c|}
\hline ARGENTINA & BOLIVIA & CHILE & $\begin{array}{c}\text { ARGENTINA, } \\
\text { BOLIVIA Y/O } \\
\text { CHILE }\end{array}$ \\
\hline \multicolumn{4}{|c|}{ Catálogo } \\
\hline \multicolumn{4}{|l|}{ Ferrario \& Galvan 1989} \\
\hline \multicolumn{4}{|l|}{ Luchini \& Verona 1972} \\
\hline \multicolumn{4}{|l|}{$\begin{array}{l}\text { Martínez-Mcchiavello \& Salas- } \\
\text { Aramburu } 1994\end{array}$} \\
\hline \multicolumn{4}{|l|}{ Tell 1985} \\
\hline \multicolumn{4}{|l|}{ Vouilloud 2003} \\
\hline \multicolumn{4}{|c|}{ Libro } \\
\hline Sar et al. 2009 & & Díaz \& Maidana 2005 & Rumrich et al. 2000 \\
\hline \multicolumn{4}{|c|}{ Artículo } \\
\hline Flower 2005 & Álvarez-Blanco et al. 2011 & Cleve-Euler 1948 & Müller 1909 \\
\hline Frenguelli 1924, 1946, 1941 & Blanco et al. 2013 & Hustedt 1927 & Krasske 1949 \\
\hline Luchini 1973 & Iltis et al. 1984 & Schreck et al. 2007 & \\
\hline Luchini 1974 & Morales \& Vis 2007 & & \\
\hline Maidana 1994 & Morales et al. 2007 & & \\
\hline Maidana \& Herbst 1989 & Morales et al. 2012 & & \\
\hline Maidana \& Seeligmann 2006 & Patrick 1961 & & \\
\hline Maidana et al. 2009 & Roux et al. 1991 & & \\
\hline Maidana et al. 2011 & Servant \& Jimenez 2001 & & \\
\hline Maidana et al. 1997 & Servant-Vildary 1978 & & \\
\hline Maidana et al. 2005 & Servant-Vildary 1984 & & \\
\hline Müller 1909 & Servant-Vildary \& Roux 1990 & & \\
\hline Seeligmann et al. 2008 & $\begin{array}{l}\text { Servant-Vildary \& Mello e } \\
\text { Souza } 1993\end{array}$ & & \\
\hline Martínez de Fabricius et al. 2003 & Sylvestre 2002 & & \\
\hline Echazú 2012 & & & \\
\hline
\end{tabular}

reportados hasta el momento exclusivamente en cuerpos de agua de altura de Argentina: Navicula atacamana (Fig. 3), N. carvajaliana (Fig. 3), N. parinacota (Fig. 4), N. phylleptosoma (Fig. 5) y $N$. salinicola var. boliviana (Fig. 5). De estos, la única especie que no es exclusivamente americana es $N$. phylleptosoma, que había sido reportada hasta el momento solo para Europa. En Argentina fue hallada en 3 humedales de altura en la provincia de Jujuy (Maidana et al., 2009).

Navicula atacamana fue descripta por Patrick (1961) para Laguna Colorada (Bolivia, 4400 $\mathrm{m} \mathrm{snm}$ ) y posteriormente fue hallada por Díaz
\& Maidana (2005) en los salares de Atacama y Punta Negra (Chile, 2300 m snm y 3000 m snm, respectivamente). Rumrich et al, (2000) la ilustran como N. spec. cf. accedens, situándola en el Parque Nacional Lauca (Chile, $4300 \mathrm{~m} \mathrm{snm}$ ).

$N$. carvajaliana, descripta por Patrick (1961) para Laguna Colorada (Bolivia, $4400 \mathrm{~m}$ snm), fue posteriormente mencionada por Iltis et al. (1984) para lagunas Honda y Cañapa (Bolivia, 3700$4500 \mathrm{~m} \mathrm{snm}$ ) y por Patrick, (1961) para la laguna Carvajal (Chile, $2400 \mathrm{~m} \mathrm{snm})$. Servant-Vildary \& Roux (1990) la destacan como dominante en lagos salinos bolivianos. 


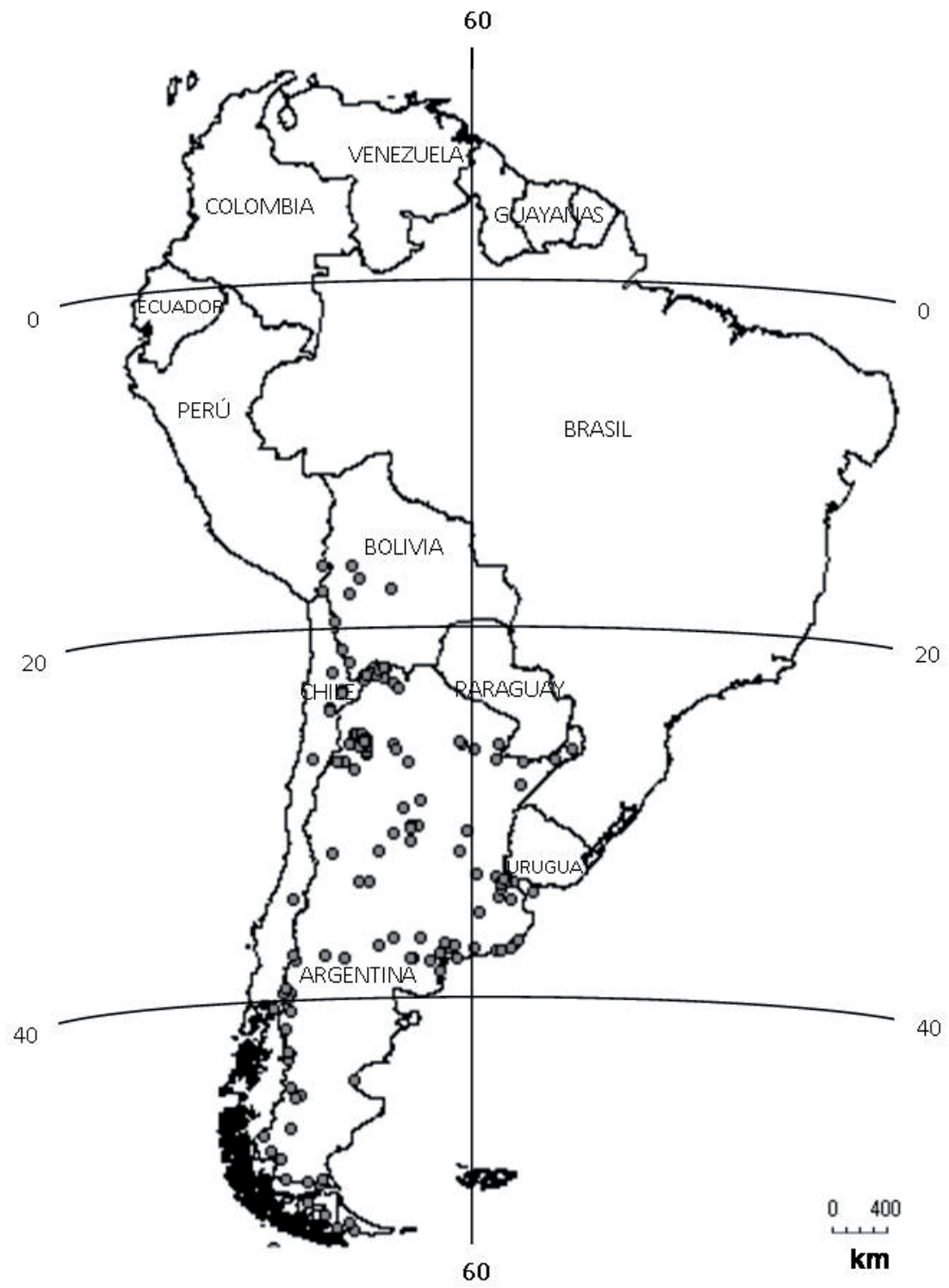

Fig. 1. Mapa de distribución de los humedales analizados para Argentina, Bolivia y Chile.

N. parinacota fue descripta por Rumrich et al. (2000) para la laguna Chungara (Chile, 4600 m snm) y citada posteriormente para los salares de Atacama y Punta Negra por Díaz \& Maidana (2005).

N. salinicola var. boliviana, que fue descripta por Patrick (1961) para Laguna Colorada (Bolivia), también fue mencionada para Chile por Díaz \& Maidana (2005).

Las cinco especies aparecieron en distintos cuerpos de agua que abarcaban un amplio rango de salinidad, según la clasificación de Cowardin et al. (1979) desde aguas dulces a hipersalinas (Fig. 6). N. parinacota, la más frecuente en los humedales de altura argentinos, se halló en ambientes francamente dulces, como Laguna Morada (Jujuy, 0,17 g/L) aunque fue más frecuente en ambientes meso hasta hipersalinos, como Laguna Diamante (Catamarca, $448 \mathrm{~g} / \mathrm{L}$ ). $N$. salinicola var. boliviana estuvo ausente en 


\begin{tabular}{|c|c|c|c|c|c|c|c|c|c|c|c|c|c|c|c|c|c|c|c|c|}
\hline & & & & & & & & ARC & GEN & ITIN & & & & & & & & & & \\
\hline & 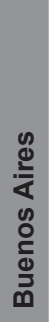 & 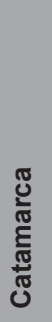 & $\begin{array}{l}\text { o } \\
\frac{\pi}{0} \\
\text { U. }\end{array}$ & 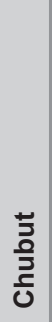 & $\begin{array}{l}\text { 을 } \\
\text { 을 } \\
\text { 잉 }\end{array}$ & 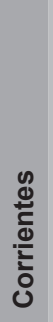 & 言 & 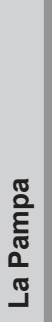 & $\begin{array}{l}\mathbb{N} \\
\frac{0}{0} \\
\frac{0}{0} \\
\frac{0}{\mathbb{J}}\end{array}$ & 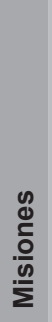 & 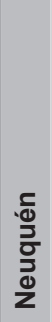 & $\begin{array}{l}\text { 응 } \\
\frac{d}{2} \\
\frac{0}{\alpha}\end{array}$ & 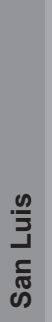 & 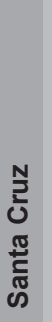 & & 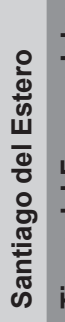 & 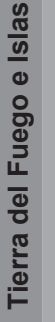 & हَ & BOLIVIA & CHILE \\
\hline Navicula atacamana & 0 & 2 & 0 & 0 & 0 & 0 & 4 & 0 & 0 & 0 & 0 & 0 & 0 & 0 & 0 & 0 & 0 & 0 & 1 & 1 \\
\hline Navicula capitatoradiata & 4 & 2 & 1 & 1 & 3 & 0 & 0 & 1 & 0 & 1 & 1 & 0 & 0 & 1 & 0 & 0 & 1 & 0 & 0 & 1 \\
\hline Navicula carvajaliana & 0 & 3 & 0 & 0 & 0 & 0 & 2 & 0 & 0 & 0 & 0 & 0 & 0 & 0 & 0 & 0 & 0 & 0 & 1 & 1 \\
\hline Navicula cari & 5 & 1 & 2 & 0 & 0 & 0 & 1 & 0 & 0 & 0 & 1 & 0 & 0 & 1 & 0 & 1 & 1 & 0 & 4 & 0 \\
\hline Navicula cincta & 5 & 9 & 1 & 0 & 2 & 0 & 2 & 0 & 0 & 0 & 1 & 0 & 1 & 3 & 1 & 1 & 1 & 0 & 4 & 1 \\
\hline Navicula cryptocephala & 5 & 2 & 1 & 0 & 5 & 1 & 4 & 1 & 1 & 0 & 3 & 4 & 1 & 1 & 1 & 1 & 2 & 3 & 5 & 5 \\
\hline Navicula cryptotenella & 4 & 8 & 2 & 0 & 1 & 0 & 13 & 1 & 0 & 0 & 0 & 3 & 0 & 2 & 1 & 0 & 2 & 2 & 3 & 0 \\
\hline Navicula cryptenelloides & 1 & 1 & 0 & 0 & 0 & 0 & 0 & 0 & 0 & 0 & 0 & 0 & 0 & 1 & 0 & 0 & 0 & 0 & 1 & 0 \\
\hline Navicula erifuga & 3 & 0 & 2 & 0 & 2 & 0 & 1 & 1 & 0 & 0 & 0 & 0 & 1 & 2 & 0 & 1 & 0 & 2 & 1 & 0 \\
\hline Navicula gregaria & 8 & 7 & 2 & 0 & 3 & 1 & 2 & 1 & 0 & 0 & 3 & 1 & 0 & 6 & 1 & 1 & 1 & 0 & 1 & 0 \\
\hline Navicula lauca & 0 & 18 & 0 & 0 & 0 & 0 & 23 & 0 & 0 & 0 & 0 & 0 & 0 & 2 & 0 & 0 & 0 & 0 & 0 & 1 \\
\hline Navicula libonensis & 0 & 15 & 0 & 0 & 1 & 0 & 8 & 1 & 0 & 0 & 0 & 1 & 1 & 4 & 0 & 0 & 0 & 0 & 1 & 0 \\
\hline Navicula microcari & 1 & 0 & 0 & 0 & 0 & 0 & 2 & 0 & 0 & 0 & 0 & 0 & 0 & 2 & 0 & 0 & 0 & 0 & 0 & 0 \\
\hline $\begin{array}{l}\text { Navicula } \\
\text { microdigitoradiata }\end{array}$ & 2 & 3 & 0 & 0 & 1 & 0 & 11 & 0 & 0 & 0 & 0 & 1 & 0 & 1 & 0 & 0 & 0 & 0 & 0 & 0 \\
\hline Navicula parinacota & 0 & 9 & 0 & 0 & 0 & 0 & 16 & 0 & 0 & 0 & 0 & 0 & 0 & 0 & 0 & 0 & 0 & 0 & 0 & 0 \\
\hline Navicula peregrina & 7 & 3 & 0 & 0 & 3 & 0 & 5 & 0 & 0 & 0 & 1 & 0 & 0 & 1 & 2 & 1 & 1 & 0 & 2 & 3 \\
\hline Navicula perminuta & 1 & 2 & 0 & 0 & 1 & 0 & 9 & 0 & 0 & 0 & 0 & 0 & 1 & 1 & 0 & 0 & 0 & 0 & 0 & 0 \\
\hline Navicula phyleptosoma & 0 & 0 & 0 & 0 & 0 & 0 & 3 & 0 & 0 & 0 & 0 & 0 & 0 & 0 & 0 & 0 & 0 & 0 & 0 & 0 \\
\hline Navicula pseudogracilis & 0 & 3 & 0 & 0 & 0 & 0 & 7 & 0 & 0 & 0 & 0 & 0 & 1 & 0 & 0 & 0 & 0 & 0 & 1 & 3 \\
\hline Navicula radiosa & 7 & 6 & 2 & 3 & 3 & 0 & 1 & 1 & 0 & 0 & 6 & 3 & 2 & 4 & 0 & 1 & 5 & 0 & 4 & 2 \\
\hline Navicula recens & 2 & 2 & 1 & 0 & 2 & 0 & 1 & 1 & 0 & 0 & 0 & 0 & 1 & 0 & 0 & 0 & 1 & 0 & 1 & 0 \\
\hline $\begin{array}{l}\text { Navicula salinicola } \\
\text { var. salinicola }\end{array}$ & 1 & 12 & 1 & 0 & 1 & 0 & 18 & 1 & 0 & 0 & 0 & 0 & 0 & 1 & 0 & 1 & 0 & 0 & 1 & 0 \\
\hline $\begin{array}{l}\text { Navicula salinicola } \\
\text { var. boliviana }\end{array}$ & 0 & 5 & 0 & 0 & 0 & 0 & 8 & 0 & 0 & 0 & 0 & 0 & 0 & 0 & 0 & 0 & 0 & 0 & 1 & 1 \\
\hline Navicula tenelloides & 2 & 4 & 1 & 0 & 2 & 0 & 1 & 1 & 0 & 0 & 2 & 1 & 0 & 2 & 0 & 1 & 1 & 0 & 1 & 1 \\
\hline Navicula tripunctata & 9 & 5 & 3 & 0 & 4 & 0 & 2 & 1 & 0 & 0 & 0 & 0 & 0 & 3 & 0 & 0 & 0 & 0 & 3 & 0 \\
\hline Navicula trivialis & 2 & 2 & 1 & 0 & 1 & 0 & 2 & 1 & 0 & 0 & 0 & 0 & 0 & 2 & 0 & 1 & 1 & 0 & 0 & 0 \\
\hline Navicula veneta & 7 & 9 & 1 & 1 & 2 & 1 & 14 & 1 & 0 & 1 & 0 & 1 & 1 & 4 & 1 & 1 & 2 & 0 & 2 & 1 \\
\hline Navicula viridula & 8 & 2 & 1 & 1 & 2 & 0 & 1 & 0 & 0 & 0 & 2 & 0 & 1 & 0 & 0 & 1 & 1 & 0 & 1 & 0 \\
\hline
\end{tabular}

aguas dulces y parece preferir las más salinas. $N$. carvajaliana se encontró solo en aguas polisalinas e hipersalinas. $N$. atacamana se halló en lagunas desde mesosalinas hasta hipersalinas. $N$. phylleptosoma apareció en una laguna de aguas dulce (Rincón de Cajas, Jujuy; 0,2 g/L), una mesosalina (Arenal, Jujuy; 4,7 g/L) y una polisalina (Catal, Jujuy; 22,5 g/L).

En cuanto a su distribución altitudinal, $N$. parinacota es la única que estuvo presente en humedales ubicados entre 2340 y $4600 \mathrm{~m}$ snm mientras que las restantes aparecieron por encima de los $3500 \mathrm{~m}$ snm (Fig. 7).

Entre las especies que, de acuerdo con la bibliografía consultada, no serían exclusivas de ambientes de altura en Argentina, N. lauca (Fig. 4), N. microcari (Fig. 4) y N. cryptotenelloides (Fig. 3) han sido reportadas en escasos lugares, 


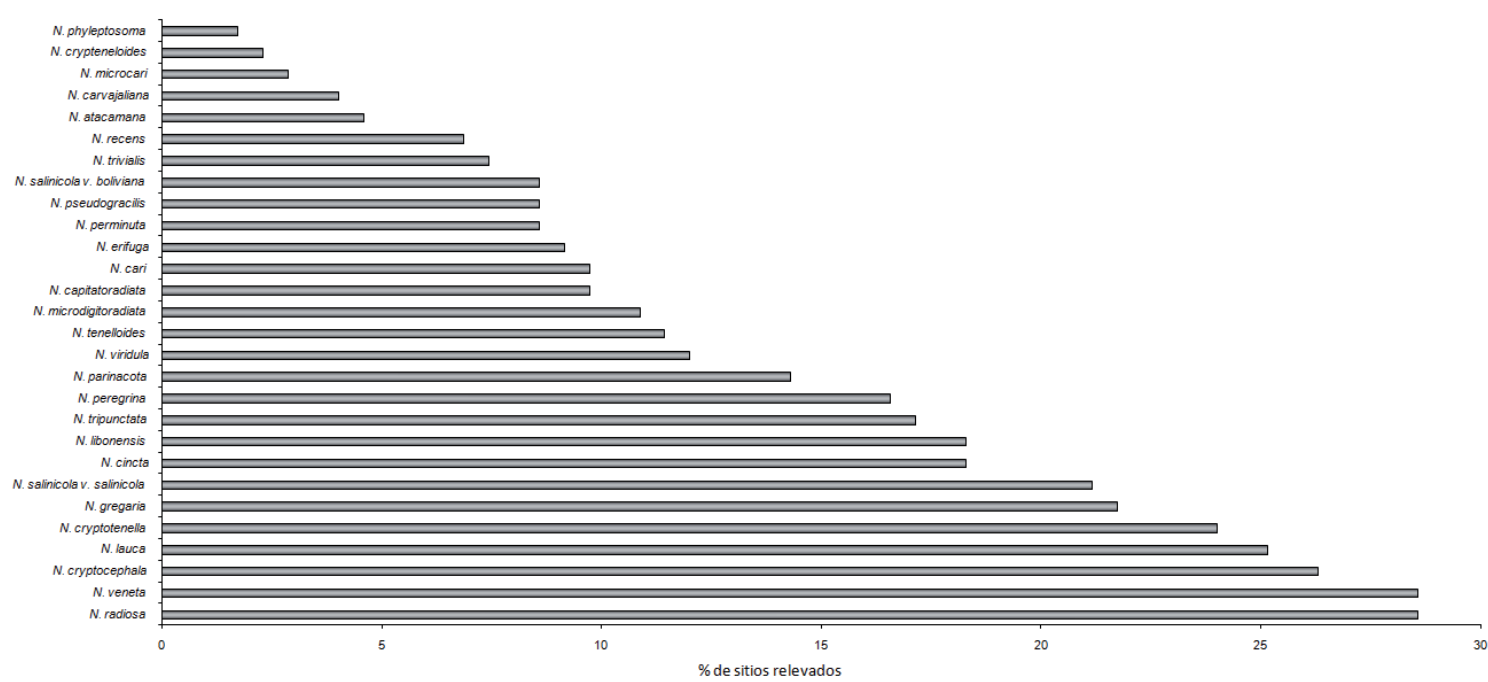

Fig. 2. Frecuencia de reportes de las 28 especies de Navicula.

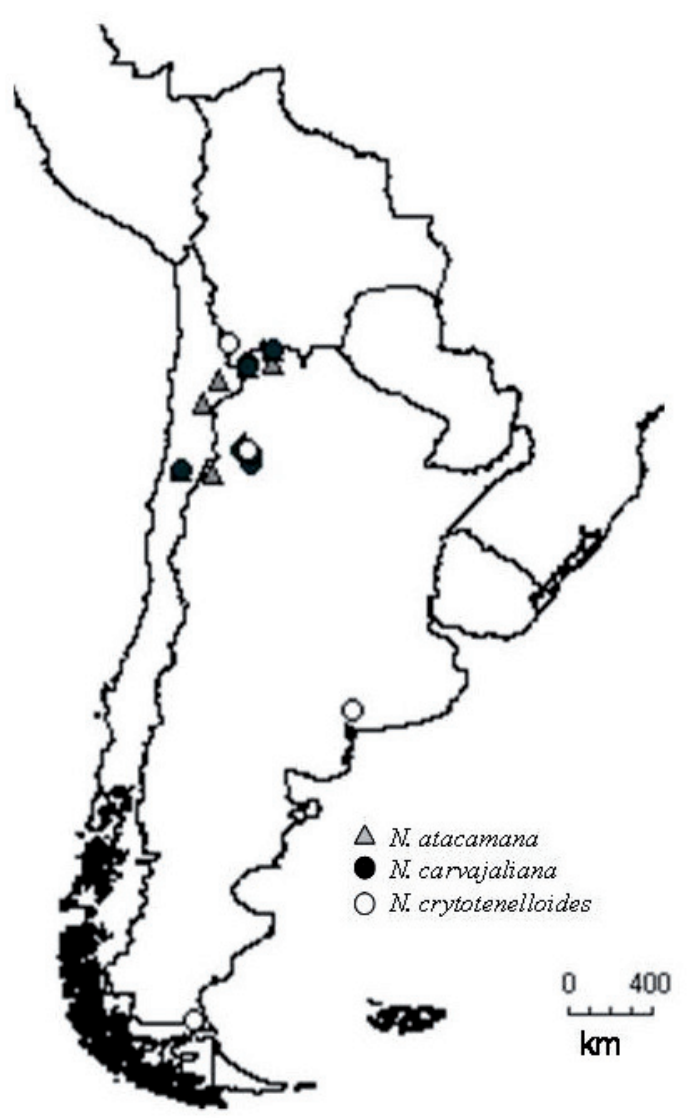

Fig. 3. Mapa de distribución de $N$. atacamana, $N$. carvajaliana y $N$. cryptotenelloides de acuerdo con la bibliografía relevada. con una distribución dispersa, tanto en ambientes de baja como alta cota.

Navicula lauca fue descripta por Rumrich et al. (2000) y hallada por ellos en el Altiplano de Chile y Bolivia y por Schreck et al. (2007) en la cuenca Cancosa (Chile), a $3950 \mathrm{~m}$ snm. En Argentina fue citada para el lago Cardiel y Laguna Honda (Santa Cruz, datos inéditos). Para el mismo país, N. microcari fue reportada en el Río Sauce Grande (Buenos Aires) y en las lagunas Potrok Aike y Chaltel (Santa Cruz; Echazú, 2012) mientras que $N$. cryptotenelloides fue mencionada para el Río Sauce Grande (Buenos Aires) y para el lago maáricoTito (Santa Cruz, datos inéditos).

Navicula perminuta Grunow in van Heurck tiene una distribución más amplia que las restantes, aunque parece estar restringida a aguas duras (Fig. 5).

Los ambientes en los cuales estuvieron representadas con mayor frecuencia las 28 especies aquí consideradas fueron, por un lado, los humedales de altura y por otro, los ambientes de baja cota pero con aguas salobres como el Río Salado (Santiago del Estero; Maidana \& Herbst, 1989) o Salinas del Bebedero (San Luis; Maidana, 1994). Sin embargo, debemos mencionar que un número elevado de las mismas especies de Navicula encontradas en humedales de altura fueron reportadas también para los ríos Luján y Cuarto (17 y 18 especies, respectivamente) que no son salinos ni transcurren por ambientes de altura. 


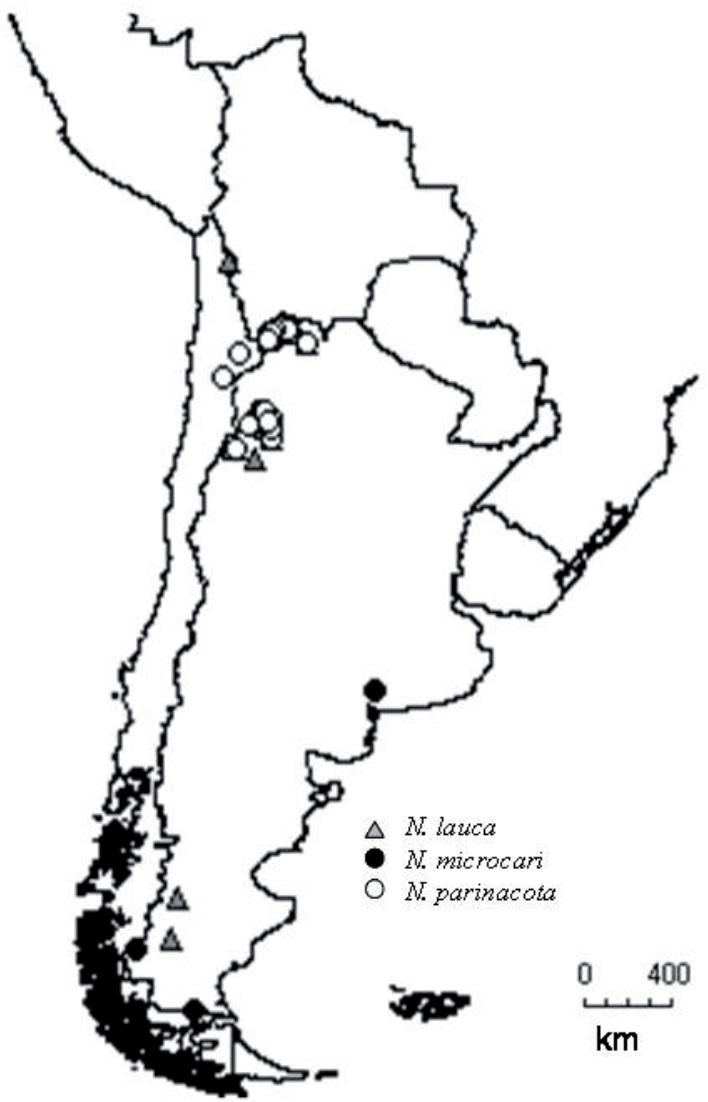

Fig. 4. Mapa de distribución de N. lauca, N. microcari y $N$. parinacota de acuerdo con la bibliografía relevada.

\section{Discusión y Conclusiones}

Round et al. (1990) señalan que Navicula es un género extremadamente común y que difícilmente pueda tomarse una muestra de epipelon sin encontrarlo. Coincidentemente con esto, en los ambientes de altura de Argentina estudiados hasta el momento, ha sido el género más frecuente y representado con el mayor número de especies. Nuestros resultados indican que una elevada proporción (82\%) de las especies de Navicula halladas en los ambientes de altura analizados son cosmopolitas o con una amplia distribución geográfica y que solo cinco son aparentemente exclusivas de humedales de alta montaña en Sudamérica (2300-4530 m snm). Existe una contradicción entre las afirmaciones de Morales (2012) y de Rumrich (2000) en cuanto al grado

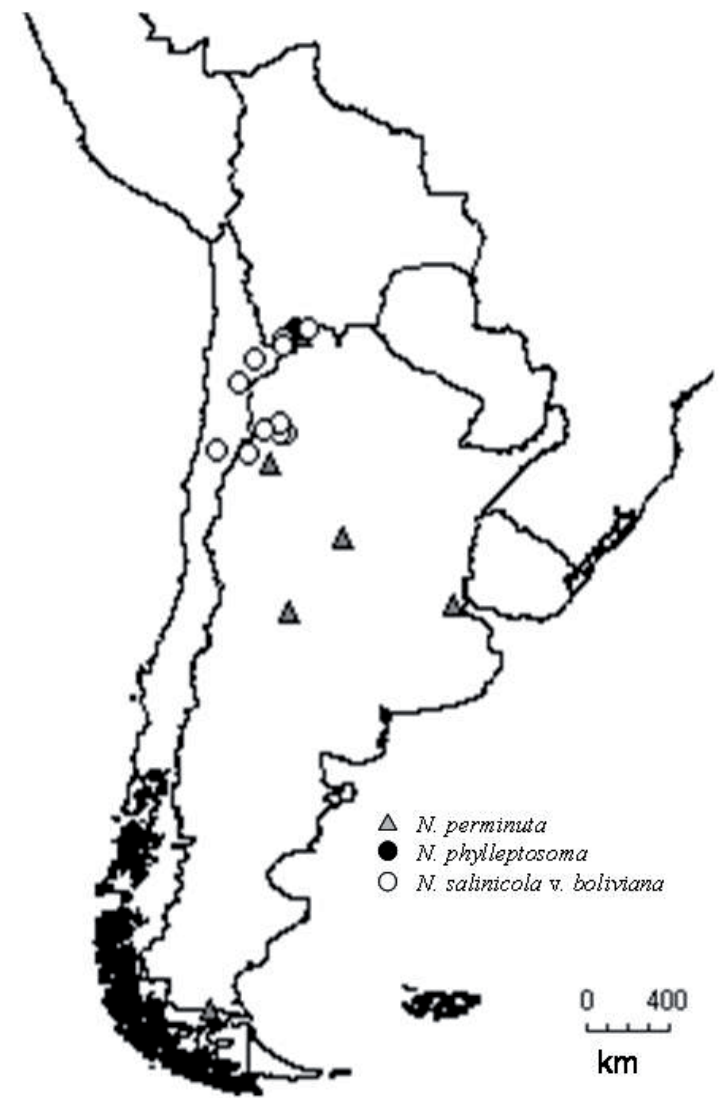

Fig. 5. Mapa de distribución de $N$. perminuta, $N$. phylleptosoma y $N$. salinicola var. boliviana de acuerdo con la bibliografía relevada.

de endemismos posibles en ambientes de alta montaña. Nuestros resultados parecerían apoyar la segunda postura, en el sentido del aparentemente bajo número de especies exclusivas encontradas pero considerando que se trata del análisis de un solo género, además cosmopolita, $18 \%$ de especies exclusivas no sería un número tan bajo.

Creemos que es posible que en muchos de los trabajos sobre diatomeas de Argentina publicados en el pasado, el alto porcentaje de especies europeas o norteamericanas reportadas sea el resultado de forzar las identificaciones para asignarles nombres de taxones conocidos. Esto fue debido a que solo desde hace relativamente poco tiempo se publicaron monografías referidas a diatomofloras sudamericanas (como las de Metzeltin \& Lange Bertalot, 1998, 2007; Metzeltin et al., 2005). Sin embargo, los 5 taxones aparentemente exclusivos 


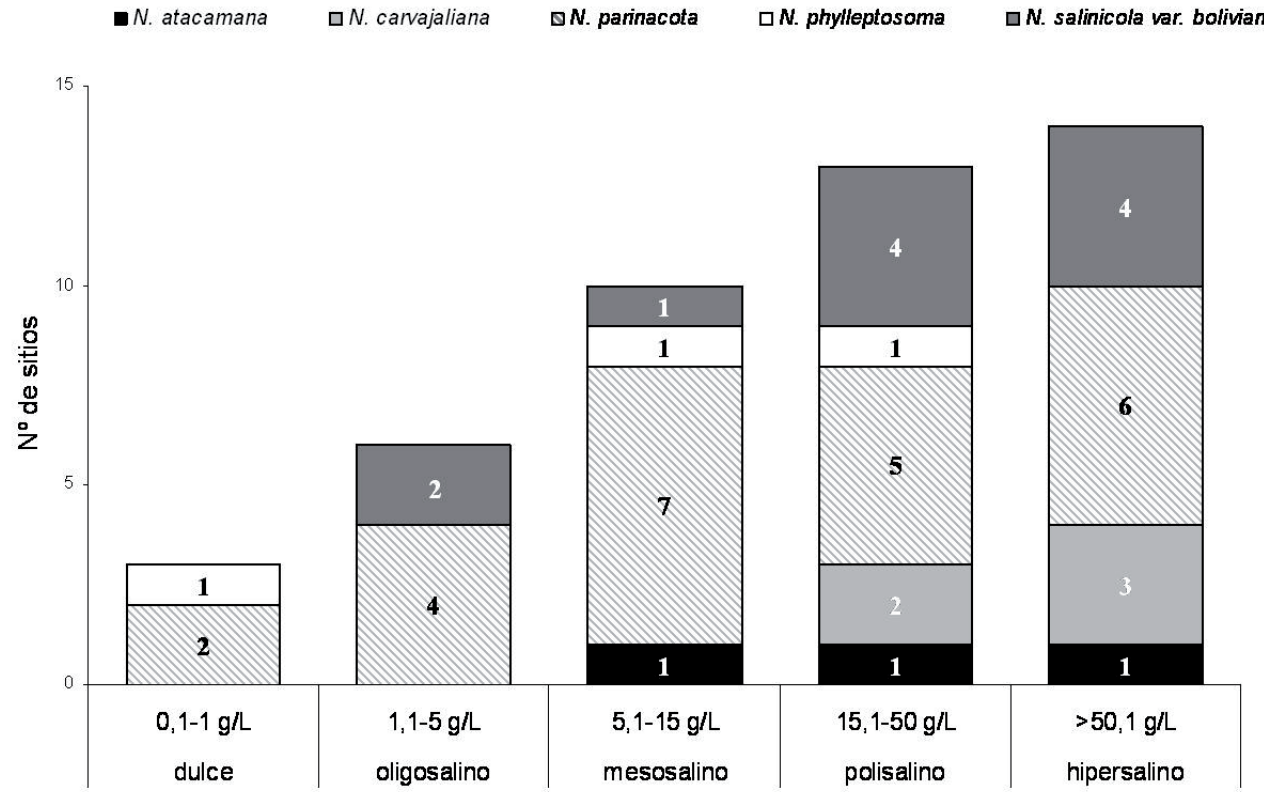

Fig. 6. Distribución de Navicula atacamana, N. carvajaliana, N. parinacota, N. phylleptosoma y N. salinicola var. boliviana, en relación a la salinidad. Los números indican la cantidad de ambientes de alta montaña para los que fueron reportadas en Argentina.

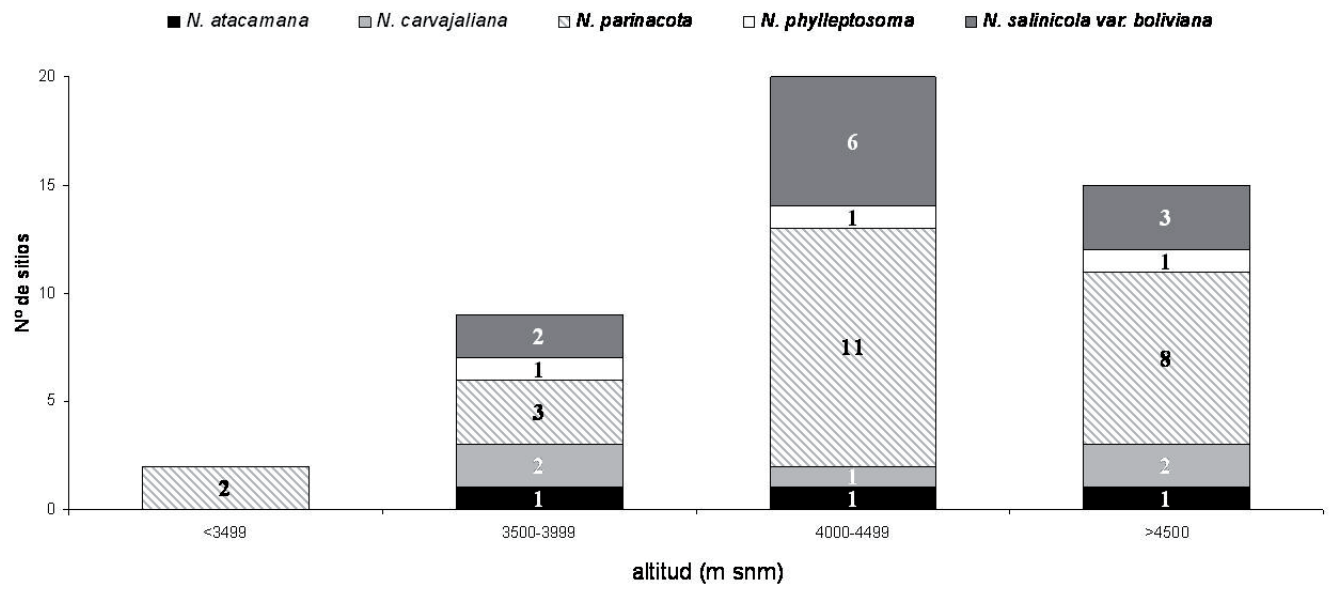

Fig. 7. Distribución altitudinal de las 5 especies de Navicula, aparentemente exclusivas de ambientes de altura en Argentina. Los números indican la cantidad de ambientes de alta montaña para los que fueron reportadas en Argentina.

en ambientes de alta montaña están incluidos entre aquellos cuya identificación pudimos corroborar. De este modo, el alto número de formas cosmopolitas en este tipo de ambientes tan particulares, solo podría atribuirse al transporte tanto natural como antrópico. Resulta llamativo que no se haya encontrado alguna especie de Navicula que sea común a todos los humedales de altura analizados, aún cuando estén muy cercanamente ubicados como es el caso del Complejo Lagunar Vilama (Maidana et al., 2009). La creciente intervención humana en la región, tanto por las actividades turísticas y deportivas 
como por las relacionadas con la industria minera, cada vez más influyentes en los ecosistemas andinos, podría traer aparejado el transporte de especies desde y hacia los humedales de altura, una de las razones por las cuales Rumrich et al. (2000) afirman que la flora andina se caracteriza por la presencia de pocas especies endémicas. Este mecanismo de dispersión, y por ende de reducción de endemismos, podría explicar el bajo número de especies propias en ambientes tales como Laguna de Los Pozuelos, que está sometida a una fuerte presión antrópica. En esta laguna, de las 15 especies identificadas de Navicula sensu stricto, luego de 3 años de muestreos estacionales, solo se halló una especie americana ( $N$. lauca, citada como $N$. sp cf. cinta var. rostrata Reimer) y las otras 14 son cosmopolitas (Maidana et al., 1997).

El resto de los cuerpos de agua estudiados se encuentran en áreas poco impactadas por lo que el factor de propagación podría ser las abundantes aves migratorias, sobre todo flamencos. Como los humedales altoandinos tienen características geológicas e hidrológicas diferentes, sólo algunas de las algas transportadas encontrarían condiciones favorables para prosperar, en función de las características físicas y/o químicas de cada cuerpo de agua y de las especies nativas residentes con las que tendrán que competir (Maidana et al., 2005).

Ninguna de las 5 especies exclusivas de altura ha sido hallada solamente en el territorio de Argentina pero, siendo esa una circunscripción hecha por el hombre, podríamos decir que, hasta ahora, estas son exclusivas de una zona geoambiental como lo serían los Andes Centrales. Sin embargo, para poder corroborarlo es necesario intensificar este tipo de estudios y extender el área analizada, abarcando el resto de los países andinos.

$N$. phylleptosoma, la única que no es exclusivamente americana entre las especies halladas solo en ambientes de altura en los sitios relevados, era conocida solo en Europa. Su hallazgo en Argentina amplía su distribución geográfica.

Es de destacar el caso de $N$. lauca, a la cual Rumrich et al. (2000) señalan como exclusiva de altura, por arriba de los $3600 \mathrm{~m}$ snm. Nuestros resultados demuestran que esto no es así ya que ha sido hallada viviendo fuera de esta región, en lagos con conductividades elevadas de la Patagonia austral argentina (laguna Potrok Aike y lago Cardiel, con 2,9 y $3,3 \mathrm{mS} / \mathrm{cm}$, respectivamente). Esta especie no parece tener una preferencia marcada en cuanto al tenor salino de las aguas ya que en los humedales de altura ha sido hallada tanto en las lagunas Diamante y Negra, de la provincia de Catamarca, que tienen una salinidad extrema de 400 y $440 \mathrm{~g} / \mathrm{L}$, respectivamente, como en las lagunas Puma-Huasi y Rincón de Cajas con 0,12 y 0,26 g/L, respectivamente. Es posible que su distribución esté relacionada con las rutas de las aves migratorias y que pudo prosperar en ambas regiones ya que tanto los humedales de altura como los patagónicos donde fue hallada tienen en común que están sometidos a una fuerte intensidad tanto de luz como de vientos y regímenes de bajas precipitaciones y temperaturas.

$\mathrm{Si}$ bien en este estudio se tuvo en cuenta un elevado número de relevamientos de la diatomoflora de Argentina, los mapas realizados muestran claramente que aún queda por estudiar una superficie importante del país. Asimismo, restan también por relevar humedales ubicados por encima de los $2000 \mathrm{~m}$ snm en los otros países sudamericanos. Este estudio representa un avance en el conocimiento de la biogeografía de las diatomeas de ambientes de altura y deja abierta la necesidad de intensificar las investigaciones que se orienten a resolver la distribución de otras diatomeas de las cuales también se ha citado un alto número de especies en este tipo de ambientes, como por ejemplo el género Nitzschia o especies de la familia Achnanthaceae.

\section{Agradecimientos}

Deseamos agradecer a la Prof. M. Brusa-Topham por su asistencia con el idioma inglés y a los dos revisores anónimos por sus valiosas sugerencias.

\section{Bibliografía}

ALONSO, R. N., B. BOOKHAGEN, B. CARRAPA, I. COUTLAND, M. HASCHKE, G. E. HILLEY, L. SCHOENBOHN, E. SOBEL, M. R. STRECKER, M. H. TRAUTH \& A. VILLANUEVA. 2006. Tectonics climate and landscape evolution of the Sothern Central Andes: The Argentina Puna Plateau and adjacent regions between 22 and $30^{\circ} \mathrm{S}$ lat. Springer Verlag, Heidelberg.

ÁlVAREZ-BLANCO, I., C. CEJUDO-FIGUEIRAS, I. DE GODOS, R. MUÑOZ \& S. BLANCO. 2011. 


\section{Seeligmann y N. I. Maidana - Navicula de ambientes de alta montaña en Argentina}

Las diatomeas de los salares del Altiplano boliviano: singularidades florísticas. Bol. R. Soc. Esp. Hist. Nat. Sec. Biol. 105: 67-82.

BLANCO, S., I. ÁlVAREZ-BLANCO, C. CEJUDOFIGUEIRAS, I. DE GODOS, E. BÉCARES, R. MUÑOZ, H. O. GUZMAN, V. A. VARGAS \& R. SOTO. 2013. New diatom taxa from high-altitude Andean saline lakes. Diatom Res. 28: 13-27.

BROWN, A. D. \& S. PACHECO. 2006. Propuesta de Actualización del Mapa Ecoregional de La Argentina. En: BROWN, A., U. MARTÍNEZ ORTIZ, M. ACERBI \& J. CORCUERA (eds.). La Situación Ambiental Argentina 2005, pp. 28-31. Fundación Vida Silvestre Argentina, Buenos Aires.

CABRERA, A. \& A. WILLINK. 1973. Biogeografía de América Latina. Programa regional de desarrollo científico y tecnológico. Secretaría General de la Organización de los Estados Americanos, Washington, D.C.

CLEVE-EULER, A. 1948. Susswasserdiatomeen aus dem Feuerland. Acta Geogr. (Soc. Geogr. Fenn.) 10: 1-61.

COWARDIN, L. M., V. CARTER, F. C. GOLET \& E. T. LAROE. 1979. Classification of wetlands and deepwater habitats of the United States. U.S. Fish and Wildlife Service, Washington, D.C.

DÍAZ, C. \& N. I. MAIDANA. 2005. Diatomeas de los salares de Atacama y Punta Negra II región. Chile. Centro de Ecología Aplicada, Santiago.

ECHAZÚ, D. M. 2012. Biodiversidad de diatomeas en humedales del sur de la Provincia de Santa Cruz, Argentina. Tesis Doctoral, Universidad de Buenos Aires.

ERICKSEN, G. E. \& R. SALAS. 1989. Geology and resources of salars in the Central Andes. In: ERICKSEN, G. E, M. T. CAÑAS PINOCHET \& J. A. REINEMUND (eds.). Geology of the Andes and its relations to hydrocarbon and mineral resource, pp. 151-164. Circum-Pacific Council for Energy and Mineral Resources, Houston, Texas.

FERRARIO, M. E. \& N. M. GALVÁN. 1989. Catálogo de las diatomeas marinas citadas entre los $36^{\circ} y$ los $60^{\circ} \mathrm{S}$ con especial referencia al mar Argentino. Dirección Nacional del Antártico, Publicación 20, Buenos Aires.

FLOWER, R. 2005. A taxonomic and ecological study of diatoms from freshwater habitats in the Falkland Islands, South Atlantic. Diatom Res. 20: 23-96.

FRENGUELLI, J. 1924. Diatomeas de Tierra del Fuego. Anales Soc. Ci. Argent. 97: 87-118, 231-266; 98: 5-63.

FRENGUELLI, J. 1926. Contribución al conocimiento de las diatomeas argentinas. IV. Diatomeas fósiles del Pelbelgranense de Miramar (Prov. de Buenos Aires). Bol. Acad. Nac. Cs. Córdoba 29: 5-89.
FRENGUELLI, J. 1941. Diatomeas del Río de La Plata. Rev. Mus. La plata (n. s.), Bot. 3: 213-334.

HUSTEDT, F. 1927. Fossile Bacillariaceen aus dem LoaBecken in der Atacama- Wüste, Chile. Archiv für Hydrobiologie 18: 224-251.

ILTIS, A., F. RISACHER \& S. SERVANT-VILDARY. 1984. Contribution á l'etude hydrobiologique des lacs salé du sud de l'altiplano bolivien. Rev. Hydrobiol. Trop. 17: 259-273.

KRASSKE, G. 1949. Subfossile Diatomeen aus den Mooren patagoniens und Feuerlands. Ann. Acad. Scient. Fennicae, Ser. A, IV Biologica 14: 1-94.

LANGE-BERTALOT, H. 2001. Diatoms of Europe 2. Navicula sensu stricto. 10 Genera Separated from Navicula sensu lato. Frustulia. A.R.G. Gantner Verlag, Ruggell.

LUCHINI, L. 1973. Contribución al estudio de la flora periférica diatómica del lago Mascardi (Provincia de Río Negro). I. Physis 32: 223-242.

LUCHINI, L. 1974. Diatomeas epífitas de algunas macrófitas del lago Situación (Prov. Chubut). Physis 33: 127-139.

LUCHINI, L. \& C. VERONA. 1972. Catálogo de las diatomeas argentinas I. Diatomeas de aguas continentales. Monogr. 2 C.I.C. Prov. de Bs. As.

MAIDANA, N. I. 1994. Fossil diatoms from Salinas del Bebedero (San Luis, Argentina). Diatoms Res. 9: 99-119.

MAIDANA, N. I. \& N. HERBST. 1989. Diatomeas (Bacilliariophyceae) de la laguna Nostra (Costumbres Calchaquies), Provincia de Tucumán, Rep. Argentina. Darwiniana 29: 63-76.

MAIDANA, N. I., M. S. VIGNA \& V. MASCITTI. 1997. Ficoflora de la laguna de Pozuelos (Jujuy, Argentina) I: Bacillariophyceae. Bol. Soc. Argent. Bot. 33: 171179.

MAIDANA, N. I., I. IZAGUIRRE, A. VINOCUR, G. MATALONI \& H. PIZARRO. 2005. Diatomeas en una transecta patagónico-antártica. Ecol. Austral 15: 159-176.

MAIDANA, N. I. \& C. SEELIGMANN. 2006. Diatomeas (Bacillariophyceae) de ambientes acuáticos de altura de la Provincia de Catamarca, Argentina II. Bol. Soc. Argent. Bot. 41: 1-13.

MAIDANA, N. I., C. SEELIGMANN \& M. MORALES. 2009. Bacillariophyceae del Complejo Lagunar Vilama (Jujuy, Argentina). Bol. Soc. Argent. Bot. 44: 257-271.

MAIDANA, N. I., C. SEELIGMANN \& M. MORALES. 2011. El género Navicula sensu stricto (Bacillariophyceae) en humedales de altura de Jujuy, Argentina. Bol. Soc. Argent. Bot. 46: 13-29.

MARTÍNEZ DE FABRICIUS, A. L., N. I. MAIDANA, N. GÓMEZ \& S. SABATER. 2003. Distribution patterns of benthic diatoms in a river exposed to 
seasonal floods: the Cuarto River (Argentina). Biodivers. Conserv. 12: 2443-2454.

MARTÍNEZ-MACCHIAVELLO, J. C. \& W. SALASARAMBURU. 1994. Catálogo Taxonómicoecológico de las diatomeas no-marinas recientes y fósiles de la República Argentina. Instituto Antártico Argentino, Publicación 27, Buenos Aires.

METZELTIN, D. \& H. LANGE-BERTALOT. 1998. Tropical diatoms of South America I: About 700 predominantly rarely known or new taxa representative of the neotropical flora. Iconogr. Diatomol. 5: 1-695.

METZELTIN, D. \& H. LANGE-BERTALOT. 2007. Tropical diatoms of South America II: Special remarks on biogeographic disjunction. Iconogr. Diatomol. 18: 1-877.

METZELTIN, D., H. LANGE-BERTALOT \& F. GARCÍA-RODRÍGUEZ. 2005. Diatoms of Uruguay; taxonomy-biogeography-diversity. Iconogr. Diatomol. 15: 1-736.

MORALES, E. A. \& M. L. VIS. 2007. Epilithic diatoms (Bacillariophyceae) from cloud forest and alpine streams in Bolivia, South America. Proc. Acad. Nat. Sci. Philadelphia 156: 123-155.

MORALES, E. A., M. L. VIS, E. FERNÁNDEZ \& P. J. KOCIOLEK. 2007. Epilithic diatoms (Bacillariophyta) from cloud forest and alpine streams in Bolivia, South America II: A preliminary report on the diatoms from Sorata, Department of $\mathrm{La}$ Paz. Acta Nova 3: 680-696.

MORALES, E., M. E. NOVAIS, G. CHÁVEZ, L. HOFFMANN \& L. ECTOR. 2012. Diatoms (Bacillariophyceae) from the Bolivian Altiplano: three new araphid species from Desagüadero River draining lake Titicaca. Fottea 12: 41-58.

MORRONE, J. J. 2006. Biogeographic areas and transition zones of Latin America and the Caribbean islands based on panbiogeographic and cladistic analyses of the entomofauna. Annu. Rev. Entomol. 51: 467-494.

MÜLLER, O. 1909. Bacillariaceen aus Südpatagoniens. Engler's botanische Jarhbücher für Systematik, Pflanzengeschichte und Pflanzengeographie 43: $1-40$.

PATRICK, R. 1961. Diatoms (Bacillariophyceae) from the alimentary tract of Phoenicoparrus jamesi (Sclater). Postilla 49: 43-55.

ROUND, F. E., M. CRAWFORD \& D. G. MANN. 1990. The Diatoms. Biology and Morphology of the genera. Cambridge University Press, Cambridge.

ROUX, M., S. SERVANT-VILDARY \& M. SERVANT. 1991. Inferred ionic composition and salinity of a Bolivian Quaternary lake, as estimated from fossil diatoms in the sediments. Hydrobiologia 210: 3-18.

RUMRICH, U., H. LANGE-BERTALOT \& M.
RUMRICH. 2000. Diatomeen der Anden von Venezuela bis Patagonien/Tierra del Fuego. Iconogr. Diatomol. 9: 1-673.

SALA, S. E. \& N. I. MAIDANA. 2003. Morphology and taxonomy of Amphora atacamae Frenguelli (Bacillariophyceae). Diatom Res. 18: 69-78.

SÁNCHEZ-CASTILLO, P., L. CRUZ-PIZARRO \& P. CARRILLO. 1989. Caracterización del fitoplancton de las lagunas de alta montaña de Sierra Nevada (Granada, España) en relación a las características físico-químicas del medio. Limnética 5: 37-50.

SAR, E. A., S. E. SALA, I. SUNESEN, M. A. HENNINGER \& M. MONTASTRUC. 2009. Catálogo de los géneros, especies y taxa infraespecificos erigidos por J. Frenguelli. Diatom monographs 10. A. R. G. Gantner Verlag K. G. Rugell, Lietchtenstein.

SCHRECK, M., N. ABARCA \& H. SCHRÖDER. 2007. Holocene sediments in the Cancosa Basin (Northern Chile): first results of diatoms analyses. Proceedings $1^{\text {st }}$ Central European Diatom Meeting: 145-149.

SEELIGMANN, C. \& N. I. MAIDANA. 2003. Diatomeas (Bacillariophyceae) en ambientes de altura de la provincia de Catamarca (Argentina). Bol. Soc. Argent. Bot. 38: 39-50.

SEELIGMANN, C., N. I. MAIDANA \& M. MORALES. 2008. Diatomeas (Bacillariophyceae) de humedales de altura de la Provincia de Jujuy - Argentina. Bol. Soc. Argent. Bot. 43: 1-517.

SERVANT, M. \& O. JIMENEZ. 2001. Holocene hydrological and climatic changes in the southern Bolivian Altiplano according to diatom assemblages in paleowetlands. Holocene 1: 267-277.

SERVANT-VILDARY, S. 1978. Les Diatomées des Sédiments superficiels d'un lac salé, Chloruré, sulfaté sodique de L'Altiplano Bolivien, le lac Poopó. Cah. ORSTOM ser. Geol. 1: 79-90.

SERVANT- VILDARY, S. 1984. Les Diatomées fluviolacustres Plio-Pleistocenes de la Formation Charaña (Cordillera Occidentale des Andes, Bolivien). Cah. ORSTOM ser. Geol. 14: 55-102.

SERVANT-VILDARY, S. \& M. ROUX, 1990. Multivariate analysis of diatoms and water chemistry in Bolivian saline lakes. Hydrobiologia 197: 267290.

SERVANT-VILDARY, S. \& S. H. MELLO E SOUSA. 1993. Palaeohydrology of the Quaternary saline Lake Ballivian (southern Bolivian Altiplano) based on diatom studies. Int. J. Salt Lake Res. 2: 69-85.

SPAULDING, S. \& D. M. MC KNIGHT. 1999. Diatoms indicators of environmental change in antarctic freshwaters. In: STOERMER, E. F. \& J. P. SMOL (eds.). The Diatoms: applications for the environmental and earth sciences, pp. 245-263. Cambridge University Press, Cambridge. 
C. Seeligmann y N. I. Maidana - Navicula de ambientes de alta montaña en Argentina

SYLVESTRE, F. 2002. A high-resolution diatom reconstruction between 21,000 and 17,000 $14 \mathrm{C}$ yr $\mathrm{BP}$ from the southern Bolivian Altiplano $\left(18-23^{\circ} \mathrm{S}\right)$. J. Paleolimnol. 27: 45-57.

TELL, G. 1985. Catálogo de las aguas de agua dulce de la República Argentina. Bibl. Phicologica 70: 1-283.

VANLANDINGHAM, S. L. 1967-1979. Catalogue of the fossil and recent genera and species of diatoms and their synonyms. I-VIII. J. Cramer, Lehre.

VANORMELINGEN, P., E. VERLEYEN \& W. VYVERMAN. 2008. The diversity and distribution of diatoms: from cosmopolitanism to narrow endemism. Biodivers. Conserv. 17: 393-405.
VOUILloud, A. 2003. Catálogo de diatomeas continentales y marinas de Argentina. Versión 1.0. En soporte magnético. Asociación Argentina de Ficología, La Plata.

Recibido el 10 de julio de 2012, aceptado el 4 de junio de 2013. 
